\title{
O FAZER DAS ENFERMEIRAS DA ESTRATÉGIA DE SAÚDE DA FAMÍLIA NA ATENÇÃO DOMICILIÁRIA
}

\author{
Cristiane Dantas Laitano LIONELLO ${ }^{a}$, Carmen Lúcia Mottin DURO \\ Andria Machado da SILVA', Regina Rigatto WITT ${ }^{\mathrm{d}}$
}

\section{RESUMO}

Este estudo objetivou conhecer o fazer das enfermeiras da Estratégia de Saúde de Família (ESF) na atenção domiciliária. Trata-se de um estudo exploratório, descritivo, com abordagem qualitativa. Foram entrevistadas seis enfermeiras atuantes da ESF de um distrito sanitário de Porto Alegre, Rio Grande do Sul, Brasil. As informações foram submetidas à análise de conteúdo temática, resultando em três categorias: a visita domiciliária (VD) na ESF, avaliação das demandas de VD e interação com a equipe de saúde na atenção domiciliária. A visita domiciliária (VD) tem oportunizado a identificação das necessidades por meio do conhecimento do contexto de vida dos usuários. Esta tem sido realizada no atendimento de doentes crônicos, acamados e idosos, efetuada a partir das informações trazidas pelos agentes comunitários de saúde. Todas as enfermeiras relataram realizar VD, sendo que gostariam de ter mais tempo para se dedicar a essa atividade.

Descritores: Enfermagem em saúde pública. Atenção primária à saúde. Assistência domiciliar.

\section{RESUMEN}

Este estudio objetivó conocer el quehacer de las enfermeras de la Estrategia de Salud de la Familia (ESF) en la atención domiciliaria. Se desarrolló un estudio exploratorio descriptivo con abordaje cualitativo. Se entrevistó a seis enfermeras que actúan en la ESF en un distrito sanitario en Porto Alegre, Rio Grande do Sul, Brasil. Las informaciones fueron sometidas al análisis de contenido temático y resutando en tres categorías: visita domiciliaria en la ESF, evaluación de las demandas de la visita domiciliaria e interacción con el equipo en la visita domiciliaria. La visita domiciliaria (VD) ha alimentado la identificación de necesidades con conocimiento del contexto de vida de los usuarios. Se la viene realizando en la atención de enfermos crónicos, acamados, ancianos, efectuada a partir de los agentes comunitarios de salud. Todas las enfermeras dicen hacer VD, pero que les gustaría más tiempo para esta actividad.

Descriptores: Enfermería en salud pública. Atención primaria de salud. Atención domiciliaria de salud. Título: El quehacer de las enfermeras de la estrategia de salud de la familia en la atención domiciliaria.

\section{ABSTRACT}

This study aimed at identifying the performance of Family Health Strategy (FHS) nurses in home care. It consists of an exploratory descriptive study using a qualitative approach. Six FHS nurses from a health district of Porto Alegre, Rio Grande do Sul, Brazil, were interviewed. Data were submitted to thematic content analysis, revealing three categories: home visit (HV) in the FHS, assessment of $H V$ demands and interaction with the health team in home care. Home visits (HV) have allowed the identification of health needs through the recognition of the patients' life context. This approach has been used for chronic, bedridden and elderly patients care, based on information brought by community health agents. All nurses reported to perform $H V$, and stated they would like to have more time to dedicate to this activity.

Descriptors: Public health nursing. Primary health care. Home care.

Title: The performance of family health nurses in home care.

a Enfermeira. Responsável Técnica do Residencial Geriátrico Vovô Francisco. Porto Alegre, Rio Grande do Sul (RS), Brasil.

b Enfermeira. Mestre em Enfermagem. Aluna do Curso de Pós-Graduação em Enfermagem da Universidade Federal do Rio Grande do Sul (UFRGS). Professora Assistente da UFRGS. Porto Alegre, RS, Brasil.

c Enfermeira. Hospital de Clínicas de Porto Alegre, Porto Alegre, RS, Brasil.

d Enfermeira. Doutora em Enfermagem em Saúde Pública. Professora Adjunta da UFRGS, Porto Alegre, RS, Brasil.

Versão on-line em Português/Inglês: http://www.scielo.br/ scielo.php?script=sci_serial\&pid=1983-1447\&lng=pt\&nrm=iso 


\section{INTRODUÇÃO}

A Estratégia de Saúde da Família (ESF) faz parte do processo de reforma do setor de saúde brasileiro, que desde a Constituição Federal de $1988^{(1)}$, visa aumentar o acesso da população ao sistema de saúde e o incremento das ações de prevenção e promoção da saúde, contribuindo para a consolidação do Sistema Único de Saúde (SUS). Os objetivos do SUS consistem na identificação e divulgação de fatores condicionantes e determinantes da saúde; na formulação de políticas de saúde destinadas a promover ações e serviços de saúde, bem como na assistência às pessoas por meio de ações de promoção, proteção e recuperação da saúde ${ }^{(2)}$.

A ESF, criada em 1994, é a estratégia prioritária do Ministério da Saúde para a organização da Atenção Básica, sendo um de seus fundamentos o acesso universal e contínuo a serviços de saúde de qualidade mediante o cadastramento e a vinculação dos usuários, reafirmando os princípios do SUS: universalização, equidade, descentralização, integralidade e participação da comunidade ${ }^{(3)}$. Esse caráter organizativo e substitutivo da ESF faz frente ao modelo tradicional de atenção básica, centrado na demanda espontânea, o que traz complexos desafios a serem superados para consolidar-se enquanto tal ${ }^{(3)}$.

As equipes básicas de trabalho que atuam na ESF são formadas por uma enfermeira, um médico, dois técnicos de enfermagem e, em média, 4 a 6 agentes comunitários de saúde. Entretanto, recentemente, foi definida uma ampliação da equipe de acordo com a realidade epidemiológica, institucional e das necessidades de saúde da população, incluindo outros profissionais como cirurgiões-dentistas, auxiliar em saúde bucal ou técnico em saúde bucal ${ }^{(3)}$.

Essa equipe deve ser capaz de planejar, organizar, desenvolver e avaliar ações que respondam às necessidades da comunidade e de articular os diversos setores envolvidos na promoção da saúde. Cada equipe de saúde da família é responsável por cerca de 1.000 famílias, ou seja, aproximadamente 4.000 pessoas $^{(3)}$.

Uma das atribuições dos profissionais de saúde na ESF é realizar o cuidado em saúde da população adscrita, no âmbito da unidade de saúde ou no domicílio $^{(3)}$. Essa proposta de atenção à saúde contrapõe-se às práticas alicerçadas na doença. A partir dessa concepção, torna-se fundamental a compreensão do contex to familiar, por meio da inserção dos profissionais de saúde no local de vida, nas interações e nas relações dos indivíduos, em sua comunidade e, principalmente, em seu domicílio ${ }^{(4)}$.

A enfermeira na ESF é responsável por realizar a assistência integral (promoção e proteção da saúde, prevenção de agravos, diagnóstico, tratamento, reabilitação e manutenção da saúde) dos indivíduos e famílias na unidade de saúde e, quando indicado ou necessário, no domicílio ${ }^{(3)}$. O domicílio é considerado um espaço de interação entre a enfermeira, a equipe de enfermagem e a família, onde o cuidado domiciliar tem como principal objetivo proporcionar a análise e revisão do desenvolvimento do processo saúde e doença $a^{(5)}$.

A atenção domiciliária é definida como um termo que envolve ações de promoção à saúde, prevenção, tratamento de doenças e reabilitação, abrangendo todas as modalidades de atendimento prestadas no domicílio, incluindo a assistência, a internação e a visita domiciliária $(\mathrm{VD})^{(6-7)}$. Quando realizada na ESF, a atenção domiciliária transpõe as práticas institucionalizadas, objetivando construir novas práticas com base na inserção dos profissionais de saúde no contexto de vida dos usuários ${ }^{(4)}$, além de propiciar a construção de vínculo e favorecer a assistência integral ${ }^{(8)}$. Apesar da amplitude de possibilidades propiciadas pela atenção domiciliária, as práticas ainda encontram-se centradas no atendimento de queixas básicas dos usuários e há falta de capacitação dos profissionais de saúde para a utilização desse espaço de atenção $0^{(5)}$. As dificuldades por parte dos profissionais, devido à estrutura ofertada para sua atuação bem como à formação profissional para a efetivação das atividades de assistência domiciliar ${ }^{(4)}$

Entende-se que, esse modo de atendimento se constitui em um espaço construtor de acesso, não apenas à assistência, mas também às políticas públicas, por meio da relação que estabelece entre os diferentes sujeitos envolvidos nesse processo. A relação entre a enfermeira e o cuidador familiar mostra que no contato entre família e equipe multiprofissional da ESF, a enfermeira merece destaque, sendo mencionada como membro referência de confiança das famílias. Os familiares recorrem à enfermeira em diferentes situações, pois sabem que ela estará disponível a ajudá-los no que estiver ao seu alcance, como momentos de angústias, incerteza 
e aflição ${ }^{(9)}$. A motivação para realizar esta investigação se deve a necessidade de conhecer como se desenvolve a atuação da enfermeira na Estratégia de Saúde da Família, tendo em vista as condições que enfrenta no seu cotidiano de trabalho. Este estudo é resultado de monografia de conclusão do curso de Enfermagem ${ }^{(10)}$ e teve como o objetivo descrever o fazer das enfermeiras da Estratégia de Saúde da Família na atenção domiciliária.

\section{MÉTODOS}

Trata-se de uma pesquisa exploratória, descritiva com abordagem qualitativa. A pesquisa qualitativa visa a compreensão da lógica interna de grupos, instituições e atores quanto a temas específicos e relações entre os indivíduos ${ }^{(11)}$.

$\mathrm{O}$ estudo foi realizado em unidades da ESF (USF) no distrito sanitário Glória/Cruzeiro/ Cristal (GCC), no município de Porto Alegre, Rio Grande do Sul, Brasil. Esse distrito apresenta em seu território 14 USF.

As participantes do estudo foram as enfermeiras atuantes nestas unidades. O critério de inclusão no estudo foi atuar nas ESF, do distrito GCC, durante o período de coleta das informações. Para o estabelecimento do número de participantes a serem entrevistados, foi utilizado o critério de saturação de dados que ocorre quando há a repetição de termos, já referidos anteriormente por outros participantes da pesquisa ${ }^{(11)}$. Assim, após a entrevista de seis enfermeiras o critério de saturação dos dados foi atingido.

Para a coleta das informações foi realizada entrevista com a questão norteadora: "fale-me sobre o seu fazer na ESF, em relação à atenção domiciliária”, com a intenção de estimular que a participante relatasse livremente a sua experiência. As entrevistas foram realizadas entre abril e maio de 2010, nas salas disponíveis nas USF, com intuito de garantir a privacidade.

Foi utilizada a análise de conteúdo do tipo temática de Minayo, que busca descobrir os núcleos de sentido, além da presença ou freqüência de fatores significativos para o objeto estudado, nos depoimentos das participantes ${ }^{(11)}$. Esta análise dividiu-se em três etapas: a pré-análise, a exploração do material, e o tratamento dos resultados obtidos e interpretação.

Inicialmente o material empírico, proveniente das entrevistas, foi transcrito e, então, realizada a leitura flutuante, buscando-se a apreensão inicial do conteúdo. Logo após, foi iniciada a leitura exaustiva do material impresso, destacando-se, nos diferentes depoimentos, os fragmentos que apresentavam semelhanças de significado. Posteriormente, na codificação e categorização de todo material empírico, procedeu-se ao processo de delimitação das unidades de registro (palavra-chave ou frase) e unidade de contexto (contexto e compreensão da unidade de registro). Este processo gerou três categorias de análise: a visita domiciliária na ESF, avaliação das demandas de visita domiciliária e operacionalização da visita domiciliária.

O projeto foi aprovado pelo Comitê de Ética em Pesquisa da Secretaria Municipal de Saúde de Porto Alegre, processo $\mathrm{N}^{\circ} 001.015 .74610 .0$. Todas as participantes assinaram o Termo de Consentimento Livre e Esclarecido. As entrevistas foram gravadas, estão arquivadas e serão destruídas após cinco anos. Para preservar a identidade dos participantes, os depoimentos foram identificados pela letra $\mathrm{E}$, seguida de números cardinais.

\section{RESULTADOS E DISCUSSÃO}

As enfermeiras que participaram do estudo referiram mais de cinco anos de experiência na ESF, porém, relataram um tempo inferior de atuação na equipe de trabalho. A partir da análise das entrevistas e de acordo com o objetivo do estudo, foram estabelecidas as seguintes categorias temáticas:

\section{A visita domiciliária na $\mathrm{ESF}$}

Ao serem perguntadas sobre seu fazer na Estratégia de Saúde da Família em relação à atenção domiciliária, as enfermeiras referiram-se à VD como uma atividade-fim, porque essa utiliza seus próprios meios para atingir um fim, isto é, o cuidado do paciente na atenção domiciliária na ESF.

Geralmente eu tenho um turno de VD que são segundas à tarde (E1).

Quanto a atenção domiciliar a gente tem agendamento todas as segundas-feiras a tarde, a minha agenda fica assim reservada só pra fazer visitas domiciliares (E2).

$A$ atenção domiciliar, eu dedico um turno na semana, que são todas as quintas de manhã (E3). 
Todas as enfermeiras se reportaram à VD como única modalidade de atenção domiciliária ${ }^{(8)}$, considerando-as como sinônimos. Porém, existem outras modalidades descritas pela literatura ${ }^{(6-7)}$. A concepção das enfermeiras do estudo apresenta-se reduzida em relação ao significado da atenção domiciliária, que envolve todas as ações que são implicadas pela assistência à saúde no domicílio e que ao serem desenvolvidas, demandam outros tipos de saberes e fazeres dos profissionais da saúde de modo a atenderem as necessidades dos usuários ${ }^{(4,8)}$. Essa concepção reduzida pode implicar na prática do enfermeiro somente contemplando a dimensão biológica, não atendendo aos pressupostos de atenção à saúde preconizados pelo modelo da ESF.

A VD oferece oportunidade para entrar em contato com o modo de vida do usuário, conhecer o ambiente e as relações intrafamiliares, abordar questões que vão além da doença física e que contemplem também os problemas sociais e emocionais, proporcionando orientações mais voltadas para as reais necessidades de saúde do usuário, e buscando singularidades na forma de cuidar ${ }^{(12)}$. Assim, o cuidado domiciliar como competência da enfermeira na atenção básica, compreende uma série de procedimentos que ao serem desempenhados possibilitam a compreensão do contex to de vida dos usuários ${ }^{(15)}$. Estes procedimentos envolvem conhecer as condições de vida do usuário e sua família para o planejamento das visitas:

Lá no ambiente em que eles vivem a gente consegue efetivar mais o nosso trabalho (E1).

A visita domiciliária é conhecer a realidade, é vivenciar, vincular melhor aquela família [...] para poder elaborar um plano de ação, porque muitas vezes o que a gente prescreve no consultório não é a realidade daquela família então no momento que tu visita, tu conhece e a tua conduta muda muito (E3).

A visita domiciliária te instrumentaliza para identificar muitas coisas dentro daquele contexto familiar e poder atuar, se inserir, implementar coisas (E6).

A VD aparece nas entrevistas como um espaço de trabalho para os profissionais na ESF, sendo utilizada como um instrumento para os cuidados em saúde na ESF e uma possibilidade de organização dos cuidados em saúde. Ao realizar a VD, o profissional utiliza uma visão diferenciada acerca do processo de adoecimento das pessoas, com maior aproximação da família e sua integração nos cuidados, possibilidade de visualizar e compreender o contex to domiciliar e nele interagir ${ }^{(12,14)}$.

As enfermeiras procuram levar a saúde para mais perto da família, a fim de melhorar a qualidade de vida dos usuários, rompendo com o modelo de atenção das unidades básicas de saúde tradicionais e estendendo suas ações para e junto à comunidade ${ }^{(4)}$. Dessa forma, as enfermeiras destacam os aspectos que devem ser levados em consideração na VD:

Eu tento ver a estrutura da moradia, isso é muito importante na hora que a gente dá as orientações aqui e tento ver o risco social dessa família, e mais os relatos dos agentes; a gente consegue fazer um diagnóstico, é o que aquela família precisa e eu acho que a visita domiciliária é isso (E1).

O conhecimento das condições do meio, como saneamento e moradia são fatores essenciais para o estabelecimento de medidas de promoção da qualidade de vida do indivíduo, famílias e comunidades, proporcionadas pela VD ${ }^{(19,15)}$. Além disso, o contexto domiciliar é uma dinâmica específica de cada casa, que abrange fatores que influenciam a vida da família, como um conjunto singular (renda, religião, crença, costume, moradia), que inclui diferentes respostas frente aos problemas apresentados, e engloba as pessoas que compartilham um mesmo ambiente de vida e de relações ${ }^{(4)}$.

Em contraposição a essa percepção da VD, alguns profissionais da ESF entendem que estas também podem consistir em ações de fiscalização de terapêuticas prescritas, conforme também já foi evidenciado por outros autores ${ }^{(12)}$. Sendo assim, as ações desenvolvidas na VD são relatadas ora como educação, ora como fiscalização:

A gente tenta educar os pacientes pra não ser uma visita domiciliária de emergência [...] (E1).

Conversar com os familiares para ver se o que o paciente está dizendo confere com o que ele está fazendo. Que nem sempre, geralmente ele diz que tá comendo, mas aquelas coisas, não tá comendo o que deveria. Tá comendo o que continua querendo comer (E5).

De acordo com o propósito da ESF, a VD deve ser realizada sob a ótica da educação em saúde, ao tornar os usuários e sua família capacitados para efetuar o seu próprio cuidado, sendo a função educadora da enfermeira imprescindível nesse processo. 
No entanto, a VD também pode significar um controle negativo sobre a vida das pessoas, ao voltar-se mais para a fiscalização, a vistoria e os registros de aspectos somente biológicos da saúde e doençca ${ }^{(16)}$.

\section{Avaliação das demandas de visita domiciliária}

$\mathrm{Na} \mathrm{ESF}$, as principais ações desenvolvidas na VD, são cadastramento de famílias, orientações, vigilância à saúde e acompanhamento de casos clínicos conforme avaliação da equipe de saúde. Além dessas ações, as enfermeiras procuram acolher a todas as demandas dos usuários, como foi expresso na fala a seguir:

A gente sempre dá um jeito, quando há uma demanda, uma prioridade a gente sempre dá um jeito de ir, mesmo não sendo naquele turno, naquele dia da semana que tá marcada a visita a gente vai no outro dia (E3).

Ao identificarem as necessidades dos usuários, as enfermeiras procuram incorporar na prática da VD o acolhimento e o acesso são diretrizes da Política de Humanização do SUS e envolve a valorização dos múltiplos aspectos que influenciam a saúde das pessoas ${ }^{(17)}$.

Um estudo indicou que as doenças de maior prevalência entre idosos atendidos por cuidados domiciliar na ESF foram: hipertensão arterial sistêmica, doença pulmonar obstrutiva crônica e diabetes melito $^{(18)}$. Corroborando com esses achados, as enfermeiras relataram que entre as demandas de VD destaca-se o atendimento de indivíduos portadores de doenças crônicas:

[...] mas a nossa demanda maisé de pacientes diabéticos crônicos, com feridas, com úlceras [...] (E4).

Os pacientes que têm doenças crônicas, esses sim a gente faz visitas regulares [...] para ver se a pessoa não piorou o estado de saúde (E5).

Muitos pacientes já são crônicos, assim a gente tem um cronograma de visita mensal (E6).

A VD de enfermagem para usuários portadores de hipertensão arterial tem se mostrado efetiva em relação à melhora na adesão ao tratamento e a outras medidas terapêuticas, além da manutenção ou diminuição do peso, da circunferência abdominal, da adesão à prática de exercícios físicos regulares e ao tratamento medicamentoso ${ }^{(19)}$. As enfermeiras realizam a VD com o propósito de verificar, fornecer e orientar o uso de medicamentos e/ou acompanhar casos clínicos consulta de enfermagem ${ }^{(18)}$, como foi evidenciado nos relatos:

Ah não tá aderindo muito ao medicamento! os agentes vão lá detectam e a gente tenta ir o mais rápido possível $[\cdots](E 1)$.

Revisar medicação é a demanda que as agentes mais trazem, revisar medicação, orientar aplicação de insulina $[\ldots](\mathrm{E} 4)$.

Essa demanda está relacionada às características demográficas da população atendida pela ESF, pois há um padrão elevado de uso de medicamentos, com pequenas variações conforme as condições de saúde entre os idosos que vivem na comunidade ${ }^{(18)}$.

Nesse contexto, encontram-se as demandas dos indivíduos acamados, com dificuldade de locomoção ou idosos, os quais apresentam necessidades especiais e que requerem a visitação domiciliar regular para supri-las:

A gente às vezes fixa aqueles que são acamados que são praticamente internados assim em casa [...] que tiveram AVC com sequelas (E4).

Eu visito mais os acamados, os pacientes idosos porque o nosso posto tem a escadaria ali que é difícil o acesso pra eles. (E2).

Os usuários acamados contam com cuidadores, que geralmente são os próprios familiares, os quais executam os cuidados, o que desperta a necessidade de educação em saúde relacionada às doenças e agravos, à terapia medicamentosa específica, às dietas e exercícios físicos pertinentes a cada situação ${ }^{(20)}$. Dessa forma, na VD as enfermeiras têm desenvolvido orientações para autonomia e responsabilização dos cuidadores na prestação do cuidado:

\section{[...] ensinar eles a fazerem pra eles ficarem quanto mais independentes melhor, (E1). \\ [...] orientar a aplicação de insulina e os curativos [...] orientações para pacientes tipo hipertensão. (E4).}

No Brasil, a maioria dos idosos que necessitam de cuidado domiciliar, não tem condições de pagar um cuidador formal. Sendo assim os 
cuidadores domiciliares de idosos geralmente são mulheres que fazem parte da família ${ }^{(20)}$. O cotidiano de um cuidador é permeado por diferentes afazeres e funções assumidas que geram uma sobrecarga tanto física quanto emocional, pois muitas vezes o cuidar de um familiar se sobrepõe a outras atividades que eram anteriormente desenvolvidas. Cientes da amplitude do cuidado domiciliar, as enfermeiras têm buscado auxiliar a capacitação da família para a prestação dos cuidados daqueles que necessitam esse tipo de atenção.

\section{Interação com a equipe de saúde na atenção domiciliária}

O trabalho da enfermeira na ESF pressupõe a articulação com outros profissionais e o envolvimento com o trabalho em equipe multidisciplinar. Os agentes comunitários de saúde (ACS) atuam tanto no intermédio das informações sobre as demandas de VD quanto na sua operacionalização:

[...] quando o agente comunitário fala: ah! o paciente piorou! ou deu algum outro problema, mesmo que eu já tenha combinado uma visita para esse paciente eu vou antes da data que eu combinei com ele (E5).

[…] geralmente essas visitas são acompanhadas pelo agente comunitário responsável pela aquela família (E3).

[...] como não tem um dia fixo a gente vai mais ou menos pela demanda das agentes comunitárias, então a cada semana surgem fatos novos e a gente então vai fazer as visitas (E4).

Na ESF, a VD é uma atividade comum a todos os profissionais da equipe multidisciplinar de saúde da família, sendo uma atribuição específica e obrigatória dos ACS. Os ACS desenvolvem a VD a partir da organização da base geográfica definida em microáreas, cadastrando e mantendo os cadastros atualizados e acompanhando todas as famílias e indivíduos sob sua responsabilidade, de acordo com as necessidades definidas pela equipe ${ }^{(3)}$. A atuação dos ACS é fundamental, pois as ações das enfermeiras e da equipe de saúde terão por base as informações dos ACS.

Participar das atividades de assistência básica sob a supervisão da enfermeira no domicílio e/ou nos demais espaços comunitários é também atribuição do técnico de enfermagem na $\mathrm{ESF}^{(3)}$. As enfermeiras exercem uma atividade de supervisão e liderança em relação ao trabalho dos agentes comunitários e técnicos de enfermagem ${ }^{(3)}$ :

[...] às vezes se precisa de uma técnica melhor, daí ou vai um técnico de enfermagem ou vai uma enfermeira, e quem avalia sou eu (E1).

O técnico visita para ver se está tudo bem com aquele paciente (E6).

A articulação com o trabalho do médico com o fazer das enfermeiras se relacionou ao atendimento dos pacientes, como os crônicos, idosos em situações precárias de vida.

[…] até porque o médico usa muito a nossa avaliação, daí ele pode ter uma ideia do que aconteceu lá (E1).

As enfermeiras relataram fazer um planejamento de visitas domiciliares, conforme demanda por ela organizada, estimando o número de visitas ou encontros para prestação da assistência domiciliar necessários para o tratamento e orientações ao usuário, bem como estabelecer a relação de ajuda à família:

\section{A gente tem todo o esquema do número de pacientes pra} poder dar atenção a todos (E2).

[...] é de acordo com o que está sendo demandado e com o nosso programa mensal (E6).

O planejamento pode ser destacado como as ações desenvolvidas em relação à demanda e à elaboração de formas de atuação tendo em vista os objetivos a serem atingidos ${ }^{(4)}$. Neste processo, as enfermeiras referem que o tempo dedicado à VD é menor do que gostariam:

[...] o tempo de visita domiciliária, de atenção domiciliar é menos do que a gente gostaria de fazer, porque o serviço burocrático do PSF é muito maior então a gente acaba não tendo tanto tempo pra fazer o que a gente gostaria, a média de visita domiciliária por mês dá o que? Umas dez visitas ... é pouco. Mas porque a gente não tem mais tempo pra fazer isso que a gente gostaria, infelizmente. (E4).

[...] a gente também tem toda a burocracia da coordenação desse posto e isso só quem faz é o coordenador então, muitas vezes, não sobra tempo como deveria pra se dedicar a visita domiciliária (E3). 
$\mathrm{Na}$ ESF, faz parte das atribuições das enfermeiras participar do gerenciamento para o adequado funcionamento da $\mathrm{USF}^{(3)}$, considerado uma competência das enfermeiras ${ }^{(13)}$. Contudo, visto que a dimensão gerencial é intrínseca ao trabalho das enfermeiras independente do contex to de atuação, é preciso desenvolver novas formas de gerenciar, que contemplem tanto atividades administrativas quanto as atividades de cuidado a saúde dos usuários. No entanto, este deve ser compartilhado com a equipe de saúde a fim de que o profissional enfermeiro disponha de tempo para realizar outras atividades, como a visita domiciliar.

\section{CONSIDERAÇÕES FINAIS}

O fazer da enfermeira da ESF na atenção domiciliária tem sido realizado por meio da VD, que permite conhecer as condições de vida dos usuários e ter uma maior compreensão das relações existentes no contexto domiciliar. Ao mesmo tempo verificou-se que a VD na ESF ainda encontra-se relacionada ao controle da adesão do usuário às prescrições da equipe de saúde, o que a reduz à dimensão biomédica da atenção à saúde necessitando de adequação a fim de atender a lógica de reorientação da atenção à saúde preconizada por este modelo de atenção à saúde.

$\mathrm{Na}$ avaliação das demandas de visita domiciliária, as enfermeiras procuram acolher as necessidades dos usuários da ESF, identificando como as principais demandas de atendimento os indivíduos com doenças crônicas, acamados, com dificuldade de locomoção e idosos. Ao realizar a VD, o foco do cuidado é o acompanhamento clínico e as atividades de educação junto aos cuidadores.

Entretanto, as enfermeiras referiram dificuldades para a realização da visita domiciliária, pois embora todas tenham relatado fazê-lo, as mesmas ressaltaram que gostariam de ter mais tempo para se dedicar a esta atividade, justificando essa impossibilidade em função da carga de atividades administrativas relacionadas ao gerenciamento das unidades de saúde que estão sob sua responsabilidade.

Este estudo limitou-se a conhecer o fazer de um dos profissionais da equipe de saúde da família. Nesse sentido, desponta a necessidade de estudos a respeito da atuação dos demais trabalhadores a fim de aprofundar a compreensão sobre esta temática na atenção básica.
Embora o estudo tenha evidenciado o fazer do enfermeiro na atenção domiciliária, este ainda tem como foco a visita domiciliar, indicando a necessidade de aprofundamento da discussão da atenção domiciliária na enfermagem, com ampliação de possibilidades do seu fazer neste âmbito.

\section{REFERÊNCIAS}

1 Brasil. Constituição da República Federativa do Brasil [Internet]. Brasília (DF); 1988 [citado 2011 Dez 27]. Disponível em: http://www.planalto.gov.br/ ccivil_03/constituicao/constitui\%C3\%A7ao.htm

2 Brasil. Lei n. 8.080, de 19 de setembro de 1990: dispõe sobre as condições para a promoção e recuperação da saúde e dá outras providências [Internet]. Brasília (DF); 1990 [citado 2011 Dez 27]. Disponível em: http://www.planalto.gov.br/ccivil_03/Leis/L8080. htm

3 Ministério da Saúde (BR). Portaria n. 2.488, de 21 de outubro de 2011: aprova a Política Nacional de Atenção Básica, estabelecendo a revisão de diretrizes e normas para a organização da Atenção Básica, para a Estratégia Saúde da Família (ESF) e o Programa de Agentes Comunitários de Saúde (PACS) [Internet]. Brasília (DF); 2011 [citado 2012 Nov 13] Disponível em: http://bvsms.saude.gov.br/bvs/saudelegis/ gm/2011/prt2488_21_10_2011.html

4. Giacomozzi CM, Lacerda MR. A prática da assistência domiciliar dos profissionais da estratégia de saúde da família. Texto \& Contexto Enferm. 2006;15(4):645-53.

5 Favero L, Lacerda MR, Mazza VA, Hermann AP. Aspectos relevantes sobre o cuidado domiciliar na produção científica da enfermagem brasileira. Rev Min Enferm. 2009;13(4):585-591.

6 Ministério da Saúde (BR), Agência Nacional de Vigilância Sanitária. Resolução RDC n. 11, de 26 de janeiro de 2006: dispõe sobre o regulamento técnico de funcionamento de serviços que prestam atenção domiciliar. Brasília (DF); 2006.

7 Lacerda MR, Giacomozzi CM, Oliniski SR, Trupel TC. Atenção à saúde no domicílio: modalidades que fundamentam sua prática. Saúde Soc. 2006;15(2):8895.

8 Santos EM, Morais SHG. A visita domiciliária na estratégia de saúde da família: percepções de enfermeiros. Cogitare Enferm. 2011;16(3):492-7. 
9 Lacerda MR, Oliniski SR. Familiares Interagindo com a enfermeira no contex to domiciliar. Rev Gaúcha Enferm. 2005;26(1):76-87.

10 Lionello CDL. O trabalho do enfermeiro da estratégia de saúde da família na atenção domiciliar no distrito Glória/Cruzeiro/Cristal-Poa-RS. [monografia]. Porto Alegre: Escola de Enfermagem, Universidade Federal do Rio Grande do Sul; 2010.

11 Minayo MCS. O desafio do conhecimento: pesquisa qualitativa em saúde. $11^{\text {a }}$ ed. São Paulo: Hucitec; 2008.

12 Sakata KN, Almeida MCP, Alvarenga AM, Craco PF, Pereira MJB.Concepções da equipe de saúde da família sobre as visitas domiciliares. Rev Bras Enferm. 2007;60(6):659-64.

13 Witt RR, Almeida MCP. Identification of nurses' competencies in primary health care through a Delphi study in southern Brazil. Public Health Nurs. 2008;25(4):335-43.

14. Persegona KR, Teixeira RC, Lacerda MR, Mantovani MF, Zagonel IPS. A dimensão expressiva do cuidado em domicílio: um despertar a partir da prática docente. Cogitare Enferm. 2007;12(3):386-91.

15 Azeredo CM, Cotta RMM, Schott M, Maia TM, Marques ES. Avaliação das condições de habitação e saneamento: a importância da visita domiciliária no contexto do Programa de Saúde da Família. Ciênc Saúde Coletiva. 2007;12(3):743-753.

16 Sossai LCF, Pinto IC. A visita domiciliária do enfermeiro: fragilidades x potencialidades. Ciênc Cuid Saúde. 2010;9(3):569-576.

17 Ministério da Saúde (BR), Secretaria Executiva. Política Nacional de Humanização: a humanização como eixo norteador das práticas de atenção e gestão em todas as instâncias do SUS. Brasília (DF); 2004.

18 Martins JJ, Silva RM, Nascimento ERP, Coelho FL, Schweitzer G, Silva RDM, et al. Idosos com necessidades de cuidado domiciliar. Rev Enferm UERJ. 2008;16(3):319-25.

19 Mantovani MF, Mottin JV, Rodrigues J. Visita domiciliária de enfermagem com atividades educativas no tratamento da pressão arterial. Online Braz J Nurs [Internet]. 2007 [citado 2011 Dez 27];6(1). Disponível em http://www.objnursing.uff.br//index.php/ nursing/article/view/757/171

20 Martins JJ, Albuquerque GL, Nascimento ERP, Barra DCC, Souza WGA, Pacheco WNS. Necessidades de educação em saúde dos cuidadores de pessoas idosas no domicílio. Texto \& Contexto Enferm. 2007;16(2):254-62.

\author{
Endereço do autor / Dirección del autor / \\ Author's address \\ Cristiane Dantas Laitano Lionello \\ Av. Doutor Carlos Barbosa, 112, Azenha \\ 90880-000, Porto Alegre, RS \\ E-mail: cristianelaitano@gmail.com
}

Recebido em: 22.01.12

Aprovado em: 28.11.12 